\title{
EL ESTILO DE ENSEÑANZA. UN CONCEPTO EN BÚSQUEDA DE PRECISIÓN
}

\begin{abstract}
Resumen
Este artículo presenta y analiza diferentes propuestas de conceptualización y clasificación de estilos de enseñanza o estilos pedagógicos. Para hacerlo puntualiza el concepto general de estilo y su sentido en el ámbito psicológico; identifica las dos tradiciones más significativas de los estudios sobre los estilos de enseñanza: la psicológica y la pedagógica; finalmente, evalúa la condición estilistica de las tipologías descritas para mantener algunas y descartar otras como ejemplares legítimos de la categoría conceptual de estilos de enseñanza.
\end{abstract}

Palabras clave: Estilo, estilo cognitivo, estilo de enseñanza, estabilidad temporal, neutralidad valorativa.

\section{THE TEACHING STYLE. SEARCHIING FOR A PRECISE CONCEPT}

\begin{abstract}
This article presents and analyzes different proposals of teaching styles' classification or pedagogical styles. To do it, the general concept of style and its sense in the psychological ambit is defined. It identifies the two most meaningful traditions in the studies of teaching styles: the psychological and the pedagogical ones; and finally, it assesses the stylistic condition of the described typologies to keep some and discard others which belong to the teaching styles category.
\end{abstract}

Key words: Style, cognitive style, teaching style, temporal stability, evaluative neutrality.

\section{INTRODUCCIÓN}

Este trabajo centra su atención en una noción que, de forma incipiente aún, ha ido ganando terreno y legitimidad en el contexto de la investigación educativa y pedagógica: la noción de estilo de enseñanza.

Para el examen de esta noción recurriremos primero al establecimiento de los orígenes de la noción general de estilo, su historia y sus características distintivas. Luego expondremos algunos de los principales planteamientos actuales de las tipologías de estilo de enseñanza y su categorización. Después, el análisis de cada una de las tipologías, desde las características de la noción ge- neral de estilo, permitirá discriminar aquellas tipologias que, en el sentido propio, no podrian ser consideradas dimensiones estilísticas. Para concluir, se establece una reflexión sobre la prospectiva y las principales necesidades de construcción de conocimiento sobre el tema.

\footnotetext{
* Doctora en Educación, profesora de la Universidad Pedagógica Nacional, Bogotá. acamargo@pedagogica.edu.co.

** Doctor en Psicologia, profesor de la Universidad Pedagógica Nacional, Bogotá. hederich@pedagogica.edu.co. Texto original recibido: 06-2-07 y aprobado: 18-4-07.
} 


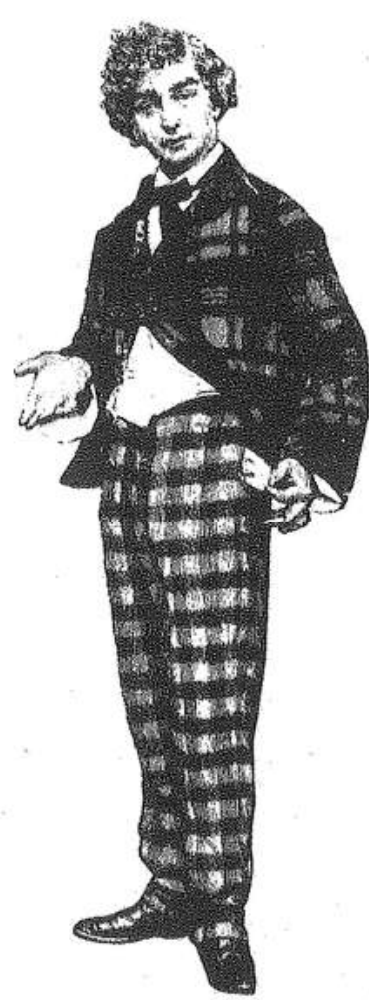

\section{LA NOCIÓN GENERAL DE ESTILO}

La noción general de estilo no tiene su origen en el entorno educativo. Esta proviene principalmente de las artes y se refiere al conjunto de características que definen una tendencia estética identificable y distintiva; bien sea de una época, por ejemplo, el estilo neoclásico, de un pueblo, el estilo africano, o de un artista, el estilo de Cervantes. Su uso tampoco está circunscrito a este ámbito de la actividad humana. Se habla también de estilos en los deportes, en la redacción de textos e incluso de estilos de desarrollo económico'. En todos los casos el término se usa para referirse a modalidades distintivas y claramente identificables en la expresión de una actividad humana.

Por extensión, el término estilo comienza a utilizarse en psicología hacia la década de 1950 para referirse a ciertos rasgos diferenciadores o individualizadores en la caracterización de una persona. Así, English e
English definen el estilo como "... la suma total de detalles de la conducta que influyen comparativamente poco en la consecución de una meta, pero que dan una manera característica, casi una identificación, a un individuo o a una actuación particular" (1958, citados por Witkin y Goodenough, 1981). En este mismo ámbito, para Messick (1987) el estilo se entiende como un conjunto de regularidades consistentes en la forma de la actividad humana que se lleva a cabo, con independencia del contenido, esto es, de los dominios propios de la actividad.

En este sentido general se han distinguido tipos de estilos diferentes relacionados con dimensiones comportamentales específicas, como los estilos expresivos (por ejemplo, Allport ${ }^{2}$, los estilos de respuesta (por ejemplo Block ${ }^{3}$ ), los estilos defensivos (por ejemplo Messick ${ }^{4}$ ), los estilos de aprendizaje (por ejemplo, Schmeck ${ }^{5}$ ) y los estilos cognitivos (por ejemplo Kogan ${ }^{6}$ ).

1 O. SUNKEL, "La interacción entre los estilos de desarrollo y el medio ambiente en América Latina". Sunkel, Oswaldo y Nocolo, Giglo en Estilos de desarrollo y medio ambiente en América Latina. México: Fondo de Cultura Económica, 1980.

${ }^{2}$ G. W. AlLPORT, Pattern and growth in personality. New York: Rineharty Winston, 1961.

3 J. BLOCK, The challenge of response sets. New York: Appleton Century Crafts, 1965.

${ }^{4} \mathrm{~S}$. Messick, Structural relationships across cognition, personality and style. En Snow, R. E. y Farr M. J. (Eds.). Aptitude learning and instruction, Vol. III. Conative and affective process analysis, 1987.

${ }^{5}$ R. R. SCHMECK, Strategies and styles of learning. An integration of varied perspectives. En Schmeck, R. R. (Ed.). Learning strategies and learning styles. New York: Plenum, 1988.

${ }^{6}$ N. Kogan, Stylistic variation in childhood and adolescence. Creativity, metaphor and cognitive style. En Flavell J. H. y Markman E.M. (Eds.). Handbook of child psychology: Cognitive development, Vol. 3. New York: Wiley, 1983.
En una nueva extensión del concepto general de estilo, el término estilo de enseñanza ha sido adoptado entre los investigadores para referirse a diferencias claramente identificables entre los profesores respecto de su forma de enseñar. Es importante aqui distinguir los elementos de forma, o manera de enseñar, de los elementos de contenido, referidos a lo que es enseñado. La noción es intuitivamente comprensible si nos remitimos a nuestra propia experiencia como alumnos: cada uno de nuestros profesores tendía a hablar de cierta forma, a plantear tipos de actividades particulares, a evaluar de una determinada manera, interactuar con cierto nivel de cercanía, etc.; en suma, cada profesor tenía cierto estilo de enseñanza característico y siempre fue tema de discusión entre los compañeros cuál era el estilo preferido de cada cual.

Sin embargo, en contraste con conceptos como el de estilo de aprendizaje, que resulta bastante claro dada la condición inequívocamente individual y distintiva del proceso mismo del aprendizaje, la noción de estilo de enseñanza ha dado lugar a diversas interpretaciones que es necesario aclarar. ¿Debe proponerse realmente un concepto como el de "estilo de enseñanza"? ¿No son suficientes las nociones que ya tenemos para describir la actividad de los profesores, como la de "enfoque pedagógico" o "perspectiva didáctica" para describir el comportamiento del profesor en el espacio del aula de clase? ¿Tiene esta noción sentido, interés o utilidad en el contexto de las investigaciones psicopedagógicas contemporáneas?

Para dar respuesta a estas preguntas, debemos examinar primero los principales trabajos que plantean estilos de enseñanza o, al menos, utilizan esta denominación específica. Una revisión de la literatura sobre el tema revela claramente la existencia de dos líneas de investigación con propósitos y desarrollos teóricos diferentes: una psicológica y otra pedagógica. A continuación, nos referiremos a ellas. 


\section{LOS ESTILOS DE ENSEÑANZA: LA TRADICIÓN PSICOLÓGICA}

La primera de las líneas de investigación sobre el estilo de enseñanza tiene un origen vinculado con la psicologia diferencial y concibe los estilos de enseñanza como una manifestación más del concepto general de estilo cognitivo. En esta tradición psicológica, el término estilo se refiere a las formas preferidas de uso de las habilidades que se poseen? Luego de varias décadas de investigación sobre los estilos, esta tradición ha propuesto diferentes rótulos, dentro de los cuales los más usados son: estilo cognitivo, estilo de aprendizaje y estilo de pensamiento. Así, en esta perspectiva, el término estilo de enseñanza "... se adopta para referirse al estilo cognitivo, de aprendizaje y de pensamiento del profesor durante su actividad docente" ${ }^{\prime 8}$. Dicho en otros términos, para esta línea psicológica, el concepto de estilo de enseñanza se define en términos de las formas preferidas de enseñar del docente que resultan connaturales a su propio estilo cognitivo.

Los primeros trabajos en esta línea psicológica se remontan a finales de la década de 1960 con el estilo cognitivo en la dimensión de independencia-dependencia de campo (DIC) ${ }^{9}$ y

\footnotetext{
${ }^{7}$ R. Sternserg, Thinking styles. New York: Cambridge University Press, 1997.

${ }^{8}$ LI-FANG ZHANG, Thinking styles. University students' preferred teaching styles and their conceptions of effective teachers. The Journal of Psychology, 138(3), 2004.

${ }^{9}$ El estilo cognitivo en la dimensión de independencia-dependencia de campo hace alusión a diferencias individuales en el tipo de información que se privilegia a la hora de llevar a cabo una tarea cognitiva. Mientras unos sujetos privilegian información interna (independientes de campo) otros privilegian información externa (dependientes de campo). En consonancia con esta descripción general, los sujetos dependientes de campo desarrollan de manera espontánea habilidades sociales, y los sujetos independientes de campo desarrollan en
}

caracterizaban las preferencias en el uso de estrategias de enseñanza por parte de profesores diferenciados por su estilo cognitivo en esta dimensión. De acuerdo con $\mathrm{Wu}^{10}$ los profesores independientes de campo favorecen procedimientos en los que puedan asumir un rol directivo impersonal. como la exposición o la disertación, frente a roles que supongan un contacto más personal con los estudiantes, que serian los preferidos por los profesores dependientes de campo. En el mismo sentido, Witkin y sus colaboradores $^{11}$, en su caracterización de las implicaciones educativas de la DIC, encontraron que mientras los profesores independientes de campo hacian un mayor énfasis en el manejo de contenidos en la clase, los profesores dependientes de campo preferian un mayor énfasis de los aspectos interpersonales de la situación de aula.

La tradición investigativa sobre tendencias estilísticas de los profesores desde la dimensión de independencia-dependencia de campo ha sido continuada recientemente por el grupo de Olivia Saracho, de la Universidad de Maryland, el cual ha trabajado principalmente la relación entre los estilos de enseñanza y los estilos de aprendizaje en el aula de clase. Saracho y sus colaboradores ${ }^{12}$ han venido construyendo un modelo descriptivo de todas las dinámicas que podrian surgir de las correspondencias o incongruencias entre los estilos cognitivos de profesores,

forma espontánea habilidades analíticas, como la capacidad de restructuración perceptual. Witkin y Goodenough; Christian Hederich y Ángela Camargo.

${ }_{10} 1967$, citado por Witkin y Goodenough, Estilos cognitivos. Naturaleza y orígenes. Madrid: Ediciones Pirámide, 1981.

${ }^{11}$ H. Witkin, C. A. Moore, D. GoOdenough yP. Cox, Field dependent and field independent cognitive style and their educational implications. Review of Educational Research, 47, 1977.

${ }^{12} \mathrm{O}$. SARACHO, Matching teachers' and students' cognitive styles. Early child development and care, 173(2-3), 2003. de estudiantes, e incluso del estilo cognitivo "implícito" en diversos contenidos de aprendizaje. Un ejemplo del tipo de investigaciones que se apoya en esta construcción teórica es el trabajo de Ford y Chen ${ }^{13}$, en el cual se examinan las implicaciones del grado de correspondencia entre el estilo cognitivo que resultaría favorecido en diferentes ambientes de aprendizaje computacional y el estilo cognitivo de los estudiantes. Esta investigación arrojó como resultado que la correspondencia favorece el logro de aprendizaje de estos últimos. La favorabilidad de la correspondencia entre estilos no es, sin embargo, completamente universal; en otros casos es la incongruencia la que favorece el logro (por ejemplo, Saracho).

Otro desarrollo similar, pero ahora dentro de otras dimensiones de estilo cognitivo: la analitico-holística y la verbal-visua/14, se destaca el trabajo de Richard Riding, de la Universidad de Birmingham, el cual ha mostrado que el estilo cognitivo del profesor se manifiesta en varios aspectos del proceso pedagógico. Específicamente, se han identificado

${ }^{13}$ N. ForD Y S. Y. CHEN, Matching/mismatching revisited. An empirical study of learning and teaching styles. British Journal of Educational Technology, 32(1), 2001.

${ }_{14} \mathrm{El}$ estilo cognitivo en la dimensión analítico-holistica constituye una reelaboración del estilo cognitivo de independencia-dependencia de campo ya presentado, y hace referencia a la tendencia de los sujetos a considerar las situaciones como compuestas por partes relativamente independientes entre sl (tendencia analítica), o bien a concebir las situaciones como un todo que debe ser mirado de manera global (tendencia holística). El estilo cognitivo verbal-visual hace referencia a las tendencias propias de la codificación de la información, bien sea de tipo verbal-conceptual o visualicónico, respectivamente. Esta dimensión ha sido desarrollada por Richard Riding durante los últimos 15 años y presenta gran desarrollo en su aplicación al sector educativo y organizacional. 
diferencias entre profesores de estilo cognitivo analítico $u$ holístico en cuanto que, mientras los profesores de estilo cognitivo holístico adoptan un estilo de enseñanza informal, los profesores de estilo analítico prefieren construir entornos de enseñanza más formales ${ }^{15}$. Así mismo, mientras los profesores de estilo holístico prefieren tratar los contenidos de aprendizaje de una manera global, pasando de un tema a otro de manera flexible, los profesores analíticos favorecen el tratamiento temático detallado, la resolución sistemática de problemas y la asignación de diferentes estructuras lógicas a un mismo contenido ${ }^{16}$. Además, mientras los profesores de estilo holístico sacan mucho provecho del trabajo en equipo y son más receptivos a las sugerencias de otros, los profesores analíticos prefieren mantener un control más estrecho de todo el proceso de enseñanza ${ }^{17}$.

Lo anterior es apenas una muestra del tipo de diferencias que profesores de uno $u$ otro estilo cognitivo manifiestan en su labor docente. Con un trabajo en la misma línea de definición de perfiles estilísticos para los profesores, Evans ${ }^{18}$ identifica un grupo de 25 aspectos de una clase que se encuentran bajo el control del profesor y, por tanto, expresan su estilo de enseñanza. Organizados según las decisiones que se toman antes o durante una clase, se mencionan, entre otras, la organización física del aula de clase, la organiza-

${ }^{15} \mathrm{~J}$. B. BigGs, What do inventories of students' learning styles really measure? A theoretical view and clarification. British Journal of Educational Psychology, 63, 1993.

${ }^{16} \mathrm{C}$. W. Allinson y J. Hayes, The cognitive style index. A measure of intuition-analysis for organizational research. Journal of Management Studies, 33, 1996.

${ }^{17} \mathrm{R}$. RIDING, School learning and cognitive style. London: David Fulton Publishers, Ltda., 2002.

${ }^{18} \mathrm{C}$. Evans, Exploring the relationship between cognitive style and teaching style. Educational Psychology, 24(4), 2004. ción de las actividades, la naturaleza de la interacción con los estudiantes, la forma de uso de los materiales, las formas de interrogar, el tipo de retroalimentación preferido frente al trabajo del estudiante y las actitudes respecto de la evaluación. Todos estos aspectos harian parte del estilo de enseñanza de un profesor con un estilo cognitivo determinado ${ }^{19}$.

En general, podría aceptarse que esta primera línea de investigación sobre los estilos de enseñanza posee una tradición firmemente respaldada por una gran cantidad de investigaciones, modelos teóricos explicativos sobre el estilo cognitivo y propuestas más bien generales de aplicación en el ámbito educativo. Una gran ventaja de esta perspectiva de trabajo sobre los estilos de enseñanza es el hecho de que aporta instrumentos sólidamente consolidados para la identificación de la tendencia cognitiva de los profesores, y por tanto de su tendencia pedagógica. Se trata de pruebas psicológicas que trabajan con indicadores de naturaleza perceptual, lo cual las hace medidas muy fuertes y confiables. Vale destacar aquí la prueba Embedded Figures Tes, EFT, para la identificación de la independencia-dependencia de campo, y la prueba Cognitive Stile Analysis, CSA, para la identificación de las tendencias analítico-holística $y$ verbal-visual.

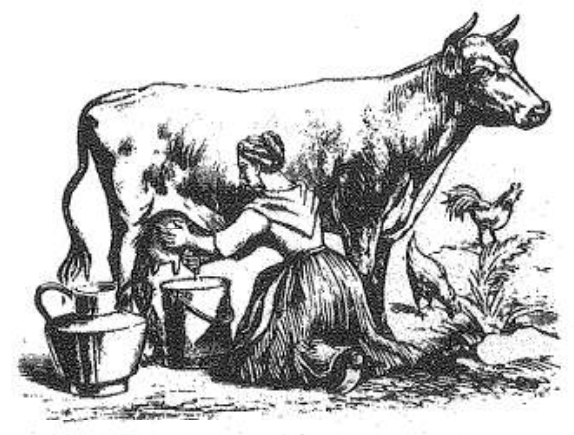

${ }^{19}$ Evans, op. cit.
En contraste con su desarrollo teórico e instrumental, la tradición de los estilos de enseñanza desde la perspectiva de los estilos cognitivos ha tenido un desarrollo más bien modesto en cuanto a trasladar tales teorías a estrategias didácticas específicas en el aula de clase. Tal y como lo afirma Evans ${ }^{20}$, a pesar de que se ha llamado la atención sobre la necesidad de mucha más investigación en ambientes de aprendizajes concretos, los estudios sobre estilos cognitivos y comportamientos de aula de profesores y alumnos son aun pocos. En este sentido, la ausencia de investigaciones sobre una enseñanza diferencial efectiva desde la tradición de los estilos cognitivos ha llevado a algunos a plantear que aun no existen caracterizaciones diferenciadoras convincentes sobre cómo enseñan los profesores ${ }^{21}$.

\section{LOS ESTILOS DE ENSEÑANZA: LA TRADICIÓN PEDAGÓGICA}

La segunda de las tradiciones intelectuales sobre el tema de los estilos de enseñanza, más claramente ligada con la investigación pedagógica, enmarca los estilos de enseñanza en el contexto de las necesidades de cualificación docente en busca de una enseñanza cada vez más efectiva, para algunos, o una enseñanza cada vez más reflexiva y consciente, para otros. En general, se utilizan diferentes criterios para establecer las clasificaciones. Veamos:

Algunos autores echan mano de atributos relacionados con la actitud más tipica del profesor cuando está frente a su clase para la formulación de sus tipologías. Bronstrom ${ }^{22}$, por ejemplo, en el contexto específico

\section{0 lbid.}

${ }^{21}$ D. Mcintyre, (1997). Teacher education in a new context. London: Paul Chapman.

${ }^{22} R$. BRONSTROM, Training style inventory. En Jones, J. L. y Pfeiffer, J. V. (Eds.). Annual handbook for group facilitatiors. San Diego: Pfeiffer, 1979. 
de la educación médica, propone una categorización de los profesores utilizando como criterio básico el énfasis que se pone en una de las posibles funciones del médico-tutor durante el proceso de enseñanza aprendizaje. Así, propone cuatro estilos: 1) el doctor, que moldea comportamientos mediante el refuerzo; 2) el experto, que hace énfasis en el conocimiento que debe adquirirse; 3 ) el entrenador, que pone su atención en la aplicación práctica del conocimiento, y 4) el humanista, que valora el autodescubrimiento en contextos acogedores y flexibles.

Muy similar a la tipologia de Bronstrom, pero esta vez en el contexto más general de la educación universitaria, es la de Grasha ${ }^{23}$. En esta tipología se propone una categorización de cinco estilos de enseñanza, que corresponden a cinco perfiles personales identificados mediante rótulos descriptivos. Son estos: 1) el experto, que confía en su profundo conocimiento de la materia que enseña; 2) la autoridad formal, que pone énfasis en su posición académica; 3 ) el modelo personal, que se plantea como un ejemplo para sus alumnos; 4) el facilitador, que valora especialmente la interacción con el estudiante, y 5) el delegador, que busca la autonomia del estudiante para el logro de su aprendizaje.

Las tipologías de Bronstrom y Grasha tienen en común el hecho de que toman en consideración las mismas dimensiones del comportamiento docente en el aula de clase: el grado de énfasis en el contenido específico a ser enseñado, la fuente de la autoridad del maestro, la naturaleza de la interacción social entre profesores y alumnos y el grado de control ejercido por el docente en la situación de clase. Al identificar los comportamientos del profesor en cada una de estas dimensiones, estas tipologías

${ }^{23}$ A. GRASHA, Teaching with style. San Bernandino, CA: Alliance Publishers, 1996. proponen rótulos que resultan ser imágenes más sugerentes que definitorias, y a las que les hace falta una descripción más sistemática de las características estructurales que las precisarian. Estos rótulos constituyen, sin embargo, aproximaciones muy valiosas al concepto de estilo de enseñanza que aún permanecen en fases iniciales de su desarrollo. Por sus características, podríamos rotular estas tipologías de estilos de enseñanza como tipologías prototípicas del rol personal del maestro.

Un segundo grupo de trabajos dentro de la tradición de la investigación pedagógica intenta caracterizar estilos pedagógicos, o de enseñanza, estableciendo la diferenciación entre unas prácticas pedagógicas consideradas tradicionales frente a otras consideradas alternativas, en algún sentido. La característica distintiva de estos trabajos es la positiva valoración que se establece, desde los planteamientos iniciales, de las prácticas alternativas. Muchos trabajos entran en este segundo grupo, entre los cuales pueden mencionarse, en el contexto internacional, el de Correia de Sousa y Santos ${ }^{23}$, que identifica dos tipos de estilos pedagógicos 0 , más bien, tipos de pedagogías sobre la base de sistemas de valores y representaciones socioculturales: el del profesor como trasmisor de conocimientos y el del profesor como facilitador de relaciones. Muy similar a esta clasificación es la de Finson y cols. ${ }^{24}$, quienes plantean estilos de enseñanza en la forma de orientaciones pedagógicas relacionadas con las concepciones del profesor sobre el área enseñada, que para este caso se trata de las ciencias naturales. Proponen entonces dos polaridades: una orientación constructivista, en la que el profesor

${ }^{24}$ L. Correia de Sousa y L. Santos, 1999. A relação entre estilos pedagógicos $e$ desempenho escolar em Portugal. Psicologia Reflexão e Crítica, 12(2). Puerto Alegre, Brasil: Universidade Federal do Rio Grande do Sul. intenta promover la búsqueda activa del conocimiento por parte del estudiante, y una orientación expositiva, en la cual el profesor se centra en la trasmisión del mismo.

Dentro de este segundo grupo de trabajos debe mencionarse, en el contexto nacional, el trabajo de Callejas y Corredor ${ }^{25}$, en el cual los estilos pedagógicos han sido conceptualizados como "... la manera propia y particular como los docentes asumen la mediación pedagógica integralmente desde su saber, saber hacer, saber comunicar y saber ser..."26. Estas dimensiones, que parecen establecidas en clara correspondencia con el concepto de competencia, les permiten identificar tres estilos pedagógicos: el del interés emancipatorio, el del interés práctico y el del interés técnico ${ }^{27}$.

En términos generales, las tipologias referenciadas antes dejan ver cierto esfuerzo por contrarrestar enfoques tradicionales de enseñanza con perspectivas basadas en teorías de enseñanza y aprendizaje más contemporáneas. Así, en vez de caracterizar la actividad concreta del profesor en el aula, intentan identificar concepciones o puntos de vista respecto de la labor educativa. De igual forma, dentro de estas tipologías de estilos de enseñanza que propenden por acciones alternativas, se intenta incorporar dentro del estilo una dimensión conceptual acerca del saber enseñado, que distingue aquellos profesores que consideran su saber como un cuerpo de cono-

${ }^{25}$ M. Callejas, y M. Corredor, (2003). La renovación de los estilos pedagógicos: colectivos para la investigación y la acción en la universidad. Revista Docencia Universitaria Cededuis, 3(1).

${ }^{26}$ L. E. SALCEDO y cols. (2005). Los estilos pedagógicos y la investigación-acción. Implicaciones en el desarrollo profesoral de los docentes universitarios. Pedagogía y Saberes, 23. Bogotá: Universidad Pedagógica Nacional.

${ }^{27}$ Callejas y Corredor, Op. cit. 


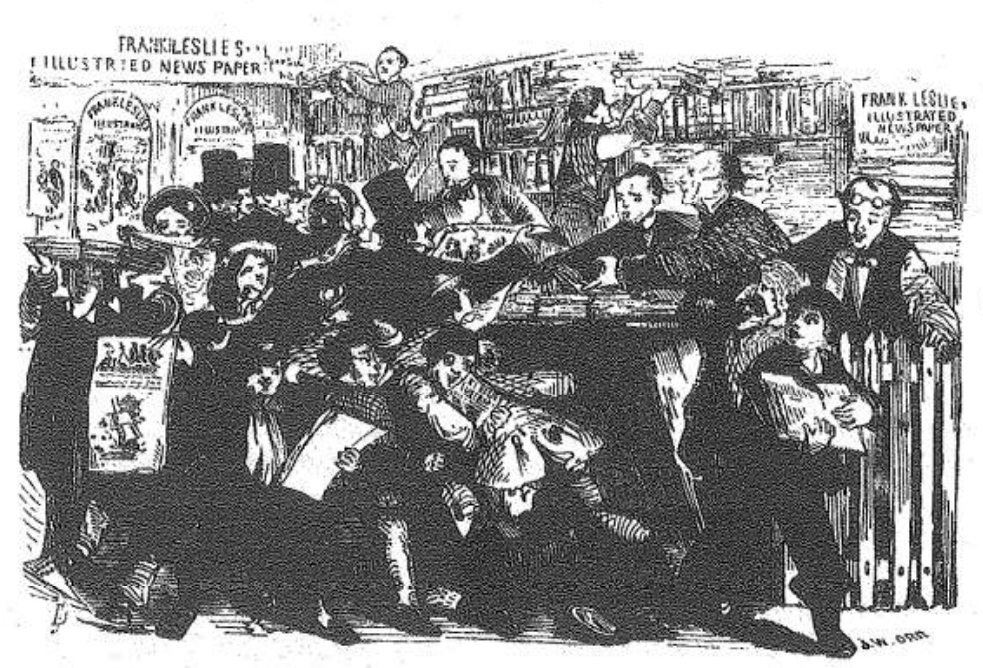

cimientos completo y acabado, de aquellos que lo consideran como algo en proceso de construcción ${ }^{28}$. Otra de las dimensiones incluidas dentro de estas tipologias es la dimensión ideológica, referida a las creencias sobre el papel social del maestro, que irían desde el maestro trasmisor-reproductor-perpetuador que mantiene el statu quo, hasta e maestro constructor-promotor-emancipador, que promueve cambios sociales.

Desde nuestro punto de vista, la inclusión de estas dimensiones relacionadas con concepciones y creencias resulta en extremo problemática para la definición del estilo de enseñanza, en la medida en que, sabemos, existe una enorme distancia entre lo que el maestro opina o cree y lo que en efecto hace en el aula de clase. ¿Hasta qué punto son los maestros consistentes con sus opiniones de la actividad pedagógica? La investigación ha mostrado que, en general, muy poco $^{29}$. En esta medida, ¿pue-

${ }^{28} \mathrm{~K}$. FINSON y cols. Comparing science teaching styles to students' perceptions of scientists. School Science and Mathematics, 106(1).

29 L. Reyes, L. Salcedo, y A. Perafan, (1999). Acciones y creencias. Tesoro oculto del educador. Bogotá: Universidad Pedagógica Nacional. den estos rasgos relacionados con las creencias y las concepciones ser definitorios de alguna dimensión estilística de la enseñanza?

Otro rasgo común a estas tipologias es el hecho de que claramente toman partido por alguna de las modalidades de enseñanza identificadas. Esto parece estar relacionado con el hecho de que cuando se enfrentan dos corrientes pedagógicas aparentemente opuestas surgen defensores radicales de cada una de ellas que impiden soluciones de compromiso ${ }^{30}$ Así, para Correia de Sousa y Santos es más deseable ser un profesor facilitador, para Finson y sus colegas es mejor tener una orientación constructivista y para Callejas y Corredor resulta más adecuado poseer un estilo pedagógico desde el interés emancipatorio. Este último rasgo resulta también bastante problemático, en tanto entra en contradicción con uno de los elementos que forman parte de la definición misma de la noción de estilo. Hablaremos de ello más adelante.
En contraste con la tradición psicológica, la línea pedagógica de estilos de enseñanza parece estar todavia en las fases iniciales de su consolidación por cuanto son pocos los modelos teóricos explicativos que ha construido y presenta menos respaldo de investigación sistemática para sus formulaciones. Igualmente, los avances de esta línea en lo que toca a los procedimientos para la evaluación del estilo de enseñanza de los profesores son aun modestos. Hasta el momento, solo se han propuesto mecanismos de autorreporte por parte del profesor (por ejemplo, Grasha $^{31}$ ) o inventarios de comportamientos de aula diligenciados por sus estudiantes (por ejemplo, Finson, 2006). Tales procedimientos pasan por alto la extrema subjetividad inherente, tanto a la evaluación de la acción propia (el profesor), como a la evaluación del comportamiento de aquel que tiene poder sobre nosotros. Diríase que esta tradición por el momento confía demasiado en las dinámicas de la presencia y la convivencia en el aula de clase.

\section{¿PUEDEN LOS ESTILOS DE ENSEÑANZA CONSIDERARSE ESTILOS?}

Una vez revisados y expuestos los trabajos más relevantes que proponen la noción de estilo de enseñan$z a$, podemos discutir la pregunta del sentido y pertinencia de la noción en el contexto de la investigación educativa y psicopedagógica.

Para hacerlo, debemos remitirnos de nuevo al concepto general de estilo que, en psicología, lleva implícitas varias características distintivas. Hederich $^{32}$ destaca cuatro que le re-

\footnotetext{
${ }^{31}$ Grasha, op. cit.

${ }^{32} \mathrm{C}$. HederICH, Estilo cognitivo en la dimensión de independencia dependencia de campo. Influencias culturales e implicaciones educativas, Tesis doctoral, Universidad Autónoma de Barcelona, 2004.
} 
sultan connaturales: 1) es una noción esencialmente diferenciadora, en la medida en que establece peculiaridades distintivas; 2 ) es relativamente estable en cada individuo; 3) es, en alguna medida, integradora de diferentes dimensiones del sujeto, y 4 ) es, en términos valorativos, neutral: no debe valorarse, en términos absolutos, un estilo por encima de otro.

Visto en estos términos, ¿en qué medida los trabajos que hemos examinado sobre los estilos de enseñanza en efecto pueden ser denominados como tales?

Iniciando con la primera de las características distintivas, ¿son los estilos de enseñanza categorias diferenciadoras del ejercicio docente? No cabe duda de que todas y cada una de las propuestas de identificación de categorías de estilos de enseñanza han sido hechas sobre la base del supuesto de la distintividad de la actividad del profesor. Se trata de una idea que, como ya lo hemos expresado, es intuitivamente inteligible y fácil de representar. En general, el solo hecho de poner el foco de la atención en la actividad del profesor $y$ de ofrecer para su actividad una serie de categorias descriptivas de su labor, representa un esfuerzo valioso frente al escaso número de trabajos actuales que intentan asignar una imagen positiva a la actividad del profesor.

Por su parte, la idea de que el estilo de enseñanza implique cierta estabilidad temporal -que corresponde a la segunda de nuestras características distintivas- la distingue de comportamientos ocasionales asociados con una situación pedagógica especifica. De acuerdo con Riding y Rayner ${ }^{34}$,

33 R. RIDING, y RAYNER, Towards a categorization of cognitive styles and learning styles. Educational Psychology, $17(1$ y 2$), 1997$.

${ }^{34} \mathrm{~L}$. CURRY, Integrating concepts of cognitive or learning styles. A review with attention to psychometric standards. Ottawa: Canadian College of Health Service Executives, 1987. es precisamente esta estabilidad temporal el rasgo más definitorio de la noción general de estilo en psicología y, a pesar de que es una característica que ha ido matizándose en el desarrollo del constructo, no ha perdido su carácter de rasgo necesario.

Al respecto de la estabilidad temporal del estilo de enseñanza vale la pena anotar que, dentro del espectro de comportamientos que despliega el maestro en la actividad de aula, deben diferenciarse algunos que dependen de factores ambientales y circunstanciales, como el nivel académico de los alumnos, el entorno físico o las características del tema enseñado, por ejemplo, de aquellos que permanecen estables por estar ligados con elementos de la personalidad del maestro, como sus modalidades de interacción social o su tolerancia frente a la ambigüedad.

Para ilustrar este punto puede evocarse el modelo de estilo cognitivo de tres capas concéntricas de Curry ${ }^{33}$, análogo a una cebolla. De acuerdo con este modelo, el estilo cognitivo del individuo tiene un centro, de carácter más bien inmutable, conformado por aspectos concernientes a los rasgos de su personalidad; a este centro le sigue una "capa" de elementos que caracterizan cierta modalidad de procesamiento de la información, que pueden variar - dentro de ciertos límites- según las características de la tarea; por último, la "cáscara de la cebolla" contiene las preferencias de aprendizaje que más varían en función de las circunstancias y las condiciones del medio ambiente. Haciendo extrapolación de este modelo, podría pensarse que el estilo de enseñanza podría tener una estructura similar, en la que existe un centro de carácter más bien permanente entre las diferentes situaciones y estable en el tiempo, y una periferia que presentaria mayor variabilidad.

Ahora bien, ¿qué tan estables son los rasgos que caracterizan las categorías de estilo de enseñanza antes presentados? Un examen detallado de las tipologías presentadas muestra que las dimensiones de estilo de enseñanza definidas desde la tradición psicológica muestran la máxima estabilidad temporal, al hacer referencia a los rasgos estructurales de la personalidad. La segunda de las tradiciones intelectuales sobre el tema, de naturaleza pedagógica, se ha mostrado menos preocupada por establecer la estabilidad del estilo de enseñanza, si bien algunos avances se vislumbran en trabajos como el de Grasha, para quien el estilo de enseñanza "... corresponde a las características y los comportamien tos personales y perdurables que surgen en la forma como conducimos nuestras clases" ${ }^{\prime 3}$. Sin embargo, en la gran mayoría de los trabajos citados desde la óptica pedagógica, y muy especialmente en aquellos que enfatizan la tensión entre pedagogías tradicionales y alternativas, no solo se ignora la estabilidad temporal del estilo de enseñanza, sino que más bien se aboga por un cambio en el mismo, cuestión que desde la primera perspectiva es difícil, además de cuestionable.

La tercera de las características mencionadas señalaría el papel del estilo como noción integradora. Esta característica diferencia la noción de estilo de la noción de rasgo, en la medida en que supone que el estilo constituye más bien un conjunto integrado de rasgos que presentan una relación funcional entre sí al ser cada uno expresión particular de la totalidad que constituye la personalidad del individuo.

Pensar que el estilo de enseñanza debe integrar diferentes rasgos del comportamiento pedagógico del profesor está en el origen de todas las tipologías presentadas, si bien no en todas se aclaran de forma suficiente las diferentes dimensiones de la actividad pedagógica que se integran en un estilo dado. Para ejemplificar este

${ }^{35}$ Grasha, op. cit., p. 1. 
punto puede mencionarse que, dentro de las tipologías de estilo de enseñanza derivadas del estilo cognitivo, en el planteamiento de independencia-dependencia de campo de Witkin se integran tres dimensiones de la actividad pedagógica relacionadas con: el manejo del contenido (estructurado-no estructurado), la forma de interacción con el estudiante (impersonal-personal) y el grado de control del profesor sobre la situación de clase (alto-bajo). Además, dentro de las tipologías derivadas de la tradición pedagógica, la de Grasha conserva la forma de interacción con el estudiante (impersonal-personal) y el grado de control del profesor sobre la situación de clase (alto-bajo) y añade la fuente de autoridad del maestro (el conocimiento-la jerarquía institucional-la función evaluadora del profesor).

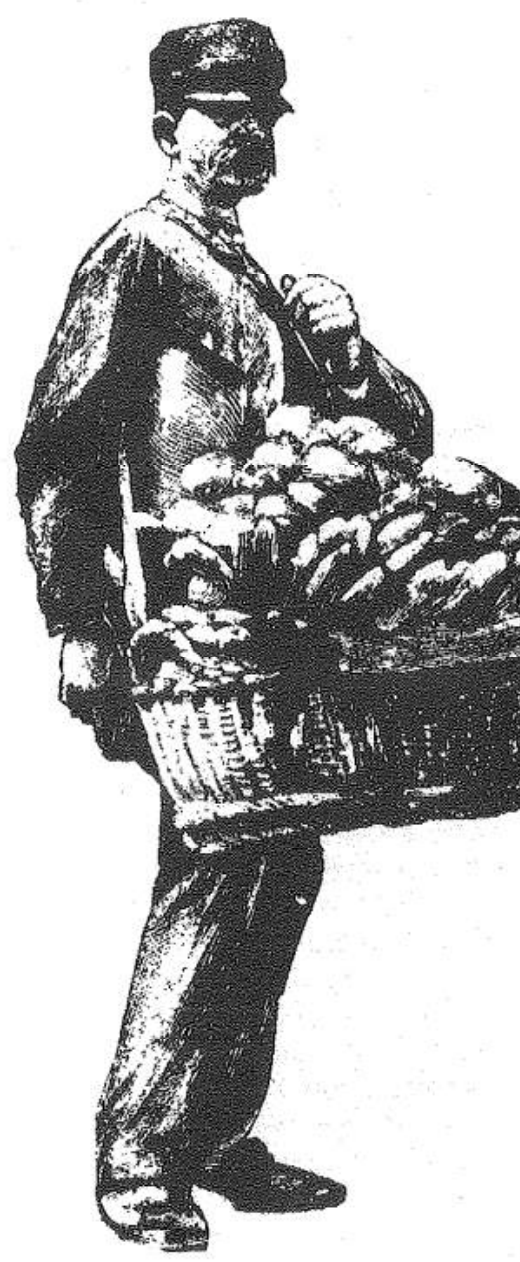

Finalmente, tenemos la caracteristica que, a nuestro juicio, es la más importante de la noción de estilo: su neutralidad valorativa. Se trata en este caso del hecho de que los valores de cualquier categoría o dimensión de estilo de la que se trate no pueden ubicarse en una escala de mejor o peor sobre la simple caracterización del valor mismo. Una cierta condición estilística solo podría evaluarse respecto de su adecuación para una cierta circunstancia (situación o tarea específicas) y nunca respecto del estilo mismo. Esta característica hace del estilo un constructo muy útil en momentos en que es necesario establecer diferencias sin abandonar ideales de equidad e imparcialidad.

Ahora bien, frente a la pregunta de si los estilos de enseñanza ya expuestos plantean categorías diferenciadoras que poseen un valor neutral, debe admitirse que algunas de las tipologías propuestas no pueden considerarse, propiamente, dimensiones de estilo de enseñanza, en tanto violan el presupuesto de la neutralidad valorativa del estilo. En efecto, a algunos de los estilos de enseñanza propuestos, y en particular a aquellos que propenden por pedagogias alternativas a las tradicionales, les subyace un sesgo valorativo explícito, en la medida en que estos trabajos plantean que es necesario mejorar ciertos estilos, o abandonar ciertos estilos en favor de otros. En estas tipologías sus autores toman partido por una de las categorías que proponen: la orientación constructivista, para Finson, el profesor facilitador para Correia de Sousa y Santos y el interés emancipatorio para Callejas y Corredor. No ocurre lo mismo con las tipologías de Grasha o Bronstrom, quienes plantean que cada estilo de enseñanza tiene una ventaja y al tiempo una desventaja, asi como en las tipologías de estilos de enseñanza de tradición psicológica. En estos casos se aboga más bien por una flexibilidad del maestro para el logro de una mejor adaptación a las características del estilo de aprendizaje de sus alumnos, aspecto que no modifica el estilo de enseñanza del docente, ni lo estigmatiza ${ }^{36}$.

Por supuesto, estas diferentes categorias de enseñanza, que no caben en la definición de estilo, poseen un valor intrínseco innegable. Su reconceptualización desde otras ópticas de la reflexión pedagógica, pero no desde la óptica de la noción de estilo, permite ubicarlos con mayor comodidad dentro de corrientes de investigación de tipo pragmático en la búsqueda de una enseñanza acorde con estándares contemporáneos ${ }^{37} \mathrm{o}$ de tipo critico reflexivo para cambiar concepciones y fortalecer la profesión docente ${ }^{38}$.

\section{ACERCA DE LA PROSPECTIVA DEL CONCEPTO}

El recorrido que hemos realizado por la literatura que de forma explícita utiliza el término estilo pedagógico o estilo de enseñanza muestra un campo de reflexión que, progresivamente, ha ido ganando terreno en la investigación pedagógica pero que, al momento presente, se encuentra en sus fases iniciales de desarrollo.

Así, se vislumbran necesidades apremiantes de desarrollo del conocimiento en el campo. En particular es necesario clarificar las dimensiones utilizadas en la definición de las tipologías estableciendo, para cada

${ }^{36}$ O. SARACHO, op. cit; Grasha, op. cit.; R. FELDER y G. Henriques, Learning and teaching styles in foreign and second language education. Foreign Language Annals, 28(1), 1965.

${ }^{37} \mathrm{~L}$. VAUGHN, y R. BAKER, Teaching in the medical setting. Balancing teaching styles, learning styles and teaching methods. Medical Teacher, 6, 2001; K. K. LEUNG y cols. Development of a teaching; M. Pressly y cols; K. Finson y cols, Comparing....

${ }^{38}$ L. Correia de Sousa y L. Santos, op. cit. 
una de ellas, sus condiciones diferenciadoras, su estabilidad temporal, su neutralidad valorativa y su co-ocurrencia con las otras dimensiones utilizadas. Estos aspectos han tenido aun poca atención por parte de los investigadores en el campo.

Otro de los campos en los que se requiere mayor investigación se relaciona con la elaboración de instrumentos que permitan la identificación del estilo de enseñanza. Aún existen demasiadas propuestas de autorreporte del profesor acerca de sus prácticas que arrojan información no muy confiable sobre las mismas. Es necesario avanzar en la construc- ción de cuestionarios dirigidos a los alumnos, así como de metodologías que permitan la triangulación de esta información. Un buen punto de inicio puede ser el trabajo de Evans y su inventario de eventos de la situación de clase que suponen decisiones del profesor $^{39}$.

Por último, debe señalarse que existe un vínculo evidente entre el estilo de enseñanza del profesor y los estilos, cognitivos o de aprendizaje, de sus alumnos; el cual explicaría en muy buena medida el logro educativo de estos últimos ${ }^{40}$. Este vínculo ha sido reconocido desde la génesis misma de algunas de las tipologías más importantes de estilo de enseñanza como la tipología de Grasha o la de Felder, que plantean de forma simultánea estilos de aprendizaje y de enseñanza. Esta representa un área de indudable interés que la consideración de estilo de enseñanza aportaría a la reflexión pedagógica. Existen, sin embargo, otras muchas áreas de desarrollo y aplicación del concepto de estilo de enseñanza. Esperamos que el presente análisis facilite el trabajo de los investigadores para su exploración y desarrollo.

\section{${ }^{39}$ EVANS, op. cit}

${ }^{40} \mathrm{O}$. SaRAcho, op. cit.

\section{BIBLIOGRAFÍA}

AlLInson, C. W. y HAYES, J., The cognitive style index. A measure of intuition-analysis for organizational research, Journal of Management Studies, 33, 1996.

ALLPORT, G. W. Pattern and growth in personality. New York: Rineharty Winston, 1961.

Biggs, J. B., What do inventories of students' learning styles really measure? A theoretical view and clarification. British Journal of Educational Psychology, 63, 1993

BLock, J. The challenge of response sets. New York: Appleton Century Crafts, 1965.

Bronstrom, R., Training style inventory. En Jones, J. L. y Pfeiffer, J. V. (Eds.). Annual handbook for group facilitatiors. San Diego: Pfeiffer, 1979.

Callejas, M. y Corredor, M. La renovación de los estilos pedagógicos: colectivos para la investigación y la acción en la universidad. Revista Docencia Universitaria Cededuis, 3(1), 2003.

CALvo, G. El aula reformada. Un análisis de las prácticas pedagógicas en la lectura y escritura a la luz de la reforma educativa. Bogotá, D. C.: UPN-CIAS.

Correia de Sousa, L. y Santos, L? (1999). $A$ relação entre estilos pedagógicos $e$ desempenho escolar em Portugal. Psicologia Reflexão e Crítica, 12(2), Puerto Alegre, Brasil: Universidade Federal do Rio Grande do Sul.

CURRY, L., Integrating concepts of cognitive or learning styles. A roviow with attention to psychometric standards. Ottawa: Canadian College of Health Service Executives, 1987.

EVANS, CAROL, Exploring the relationship between cognitive style and teaching style. Educational Psychology, 24(4), 2004.

Felder, R. y Henriques, E., Learning and teaching styles in foreign and second language education. Foreign Language Annals, 28(1), 1965.

Finson, K., Thomas, J. y Pedersen, J., Comparing science teaching styles to students' perceptions of scientists. School Science and Mathematics, 106(1), 2006.

FORD, N. y CHEN, S. Y., Matching/mismatching revisited. An empirical study of learning and teaching styles. British Journal of Educational Technology, 32(1), 2001.

Friedman, P. y AlLey, R., Learning/teaching styles. Applying the principles. Theory into Practice. XXIII(1), 1982.
GrASHA, A., Teaching with style. San Bernandino, CA: Alliance Publi-shers, 1996.

HEDERICH, C., Estilo cognitivo en la dimensión de independencia dependencia de campo. Influencias culturales e implicaciones educativas, Tesis doctoral, Universidad Autónoma de Barcelona, 2004.

Hederich, C. y Camargo, Á., Estilos cognitivos como modalidades de procesamiento de la información. Bogotá: Colciencias-Universidad Pedagógica Nacional, 1999.

Kogan, N., Stylistic variation in childhood and adolescence. Creativity, metaphor and cognitive style. En Flavell J. H. y Markman E.M. (Eds.). Handbook of child psychology: Cognitive development, 3. New York: Wiley, 1983.

Leung, Kal-Kuen, Lue, Bee-Horng y Lee, Ming-BeEn, Development of a teaching style inventory for tutor evaluation in problem-based learning. Medical Education, $37,2003$.

MCINTYRE, D., Teacher education in a new context. London: Paul Chapman, 1997.

MEssick, S., Structural relationships across cognition, personality and style. En Snow, R. E. y Farr M. J. (Eds.). Aptitude learning and instruction, Vol. III. Conative and affective process analysis, 1987. 
Montauk, S. y Grasha, A., Adult HIV outpatient care. A handbook for clinical teaching. Cincinnatti: University of Cincinnatti. Department of Family Medicine, 1993.

Pressley, M., Röehrig, A., Raphael, L., Dolezal, S., BOHN, C., MOHAN, L., WhaRTON-MCDONALD, R. BOGNER, K. Y HOGAN, K. (2003). Teaching processes in elementary and secondary education. En Reynolds, W. y Miller, G. (Eds.). Handbook of Psychology, 7. Educational psychology, Hoboken, New Jersey: John Willey and Sons, Inc.

Reyes, L., Salcedo, L. y Perafan, A. (1999). Acciones y creencias. Tesoro oculto del educador. Bogotá: Universidad Pedagógica Nacional.

RIDING, R., School learning and cognitive style. London: David Fulton Publishers, Ltda., 2002.

Riding, R. y CheEma, I., Cognitive styles. An overview and integration. Educational Psychology, 11(3-4), 1991.
RIDING, R. y RAYNER, Towards a categorization of cognitive styles and learning styles. Educational Psychology, 17(1-2), 1997.

SAdLeR-Smith, E. y Riding, R., Cognitive styles and learning instructional preferentes. Instructional Science, 27, 1999.

Salcedo, L. E.; Forero, F; Callejas, M. M.; PARDO, A. y OVIEDO, P. E., Los estilos pedagógicos y la investigación-acción. Implicaciones en el desarrollo profesoral de los docentes universitarios. Pedagogía y Saberes, N. ${ }^{\circ} 23$. Bogotá: Universidad Pedagógica Nacional, 2005.

SARACHO, O., Matching teachers' and students' cognitive styles. Early child development and care, 173(2-3), 2003.

SCHMECK, R. R., Strategies and styles of learning. An integration of varied perspectives. En Schmeck, R. R. (Ed.). Learning strategies and learning styles. New York: Plenum, 1988.
SUNKEL, O., "La interacción entre los estilos de desarrollo y el medio ambiente en América Latina". Sunkel, Oswaldo y Giglo, Nocolo en Estilos de desarrollo y medio ambiente en América Latina. México: Dondo de Cultura Económica, 1980.

STERnBerg, R., Thinking styles. New York: Cambridge University Press, 1997.

VAUGHN, L. y BAKER, R., Teaching in the medical setting. Balancing teaching styles, learning styles and teaching methods. Medical Teacher, 6, 2001.

WitKIN, H. y GOODENOUGH, D., Estilos cog nitivos. Naturaleza y orígenes. Madrid: Ediciones Pirámide, 1981.

Witkin, H., Moore, C. A., Goodenough, D. y Cox, P., Field dependent and field independent cognitive style and their educational implications. Review of Educational Research, 47, 1977.

ZHANG, LI-FANG, Thinking styles. University students' preferred teaching styles and their conceptions of effective teachers. The Journal of Psychology, 138(3), 2004 